\title{
Coexistence of tuberculous gumma with tuberculosis verrucous cutis (TBVC) in an immunocompetent female
}

\author{
Nishant Agarwala, Madhuchhanda Mohapatra, Trashita Hassanandani, Maitreyee Panda
}

Department of Dermatology Venerology and Leprology, IMS and SUM Hospital, Bhubaneswar, Odisha, India

Corresponding author: Dr. Trashita Hassanandani, E-mail: htrashita@gmail.com

\begin{abstract}
Tuberculous gummas and tuberculosis verrucosa cutis (TBVC) generally manifest in two extreme poles across the cell mediated immunity spectrum of cutaneous tuberculosis. The present case report refers to a 43 year old female with subcutaneous soft to firm non-tender, minimally fluctuant nodules \& abscesses over digits of B/L hands and few well defined verrucous plaques over lateral aspects of bilateral soles. Ziehl-Neelsen stain did not demonstrate any acid fast bacilli. On histopathologic examination it was diagnosed as Tuberculous gumma with Tuberculosis Verrucosa cutis as per clinical diagnosis and its coexistence in a single individual is unique. The patient was treated with anti tubercular drugs (ATD) \& responded well.
\end{abstract}

Key words: Cutaneous tuberculosis; Tuberculous gumma; Tuberculosis verrucous cutis (TBVC); Immunocompetent

\section{INTRODUCTION}

Cutaneous tuberculosis constituting a small fraction about $1.5 \%$ of all forms of extra pulmonary tuberculosis, manifests as different clinical types across the cell mediated immunity spectrum. The clinical manifestations like lupus vulgaris, tuberculosis verrucosa cutis (TBVC), scrofuloderma, tuberculous gumma, tuberculous chancre, miliary tuberculosis, papulonecrotic tuberculid and lichen scrofulosorum may be dependent on the route of infection (endogenous or exogenous), the immune status of the patient and previous sensitization with tuberculosis [1]. Tuberculous gummas, also called metastatic tuberculous abscesses, are usually seen in malnourished children and in immunocompromised states, as result of hematogenous dissemination of tubercle bacilli from an underlying focus and occur during periods of lowered immunity [2]. It usually presents as one or multiple non-tender subcutaneous nodules, which slowly soften, or cold abscesses with fluctuant swelling. Whereas TBVC results from direct inoculation of the bacilli into the skin of previously infected patients having intact immunity. It manifests as a large verrucous plaque with finger like projections at the margins. Co-existence of different types of cutaneous tuberculosis has been described in various literatures in the form of scrofuloderma with tuberculosis verrucosa cutis [3], lupus vulgaris with tuberculosis verrucosa cutis, papulonecrotic tuberculid with lichen scrofulosorum and lupus vulgaris with papulonecrotic tuberculid [4]. Interestingly, we came across a rare presentation in a 43 year immunocompetent female presenting with both subcutaneous skin abscesses in multiple sites (low immunity) along with verrucous plaques over B/L feet (high immunity), proven histopatholigically as TB gumma and TBVC respectively.

\section{CASE REPORT}

A 43 year old female presented to us with chief complaints of multiple painless swellings over digits of $\mathrm{B} / \mathrm{L}$ hands for 4 weeks. On examination, there were multiple subcutaneous soft to firm non-tender, minimally fluctuant nodules \&

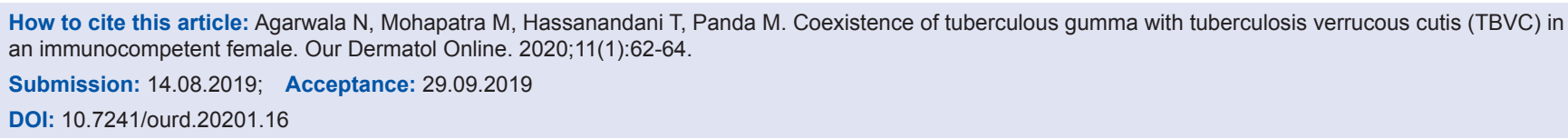


abscesses of size about $3 * 3 \mathrm{~cm}^{2}$ with overlying intact skin, mild erythema and no surface change (Fig. 1). On further examination, we found few well defined verrucous plaques over lateral aspects of bilateral soles which was persisting since 2 months (Fig. 2). We sent samples from both lesions for histopathological study. Routine blood investigations were within normal limits, except raised ESR (68cm/hour). Except for episodic mild raise of temperature (99-100F) patient had no other systemic complaints. There was no evidence of any immunosuppression or lymphadenopathy. Radiological investigations for bony involvement were negative. Mantoux test was positive with $15 \mathrm{~mm}$ induration at 48 hours. Gram stain and Ziehl-Neelsen stain for acid fast bacilli were negative. PCR for Mycobacterium tuberculosis was negative. HP study from the nodules showed multiple granulomas composed of epitheloid cells, histiocytes, lymphocytes and Langhan's type of giant cells with small areas of caseous necrosis involving reticular dermis and subcutaneous tissue (Figs. 3 a and 3b). The verrucous lesions on HP study revealed mildly hyperkeratotic epidermis with well-defined granulomas

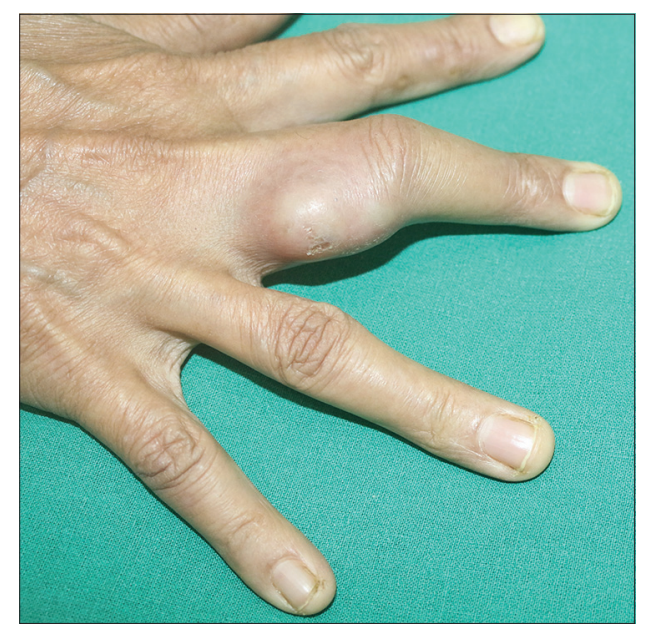

Figure 1: A skin coloured, soft to firm, fluctuant nodule on the right middle finger.

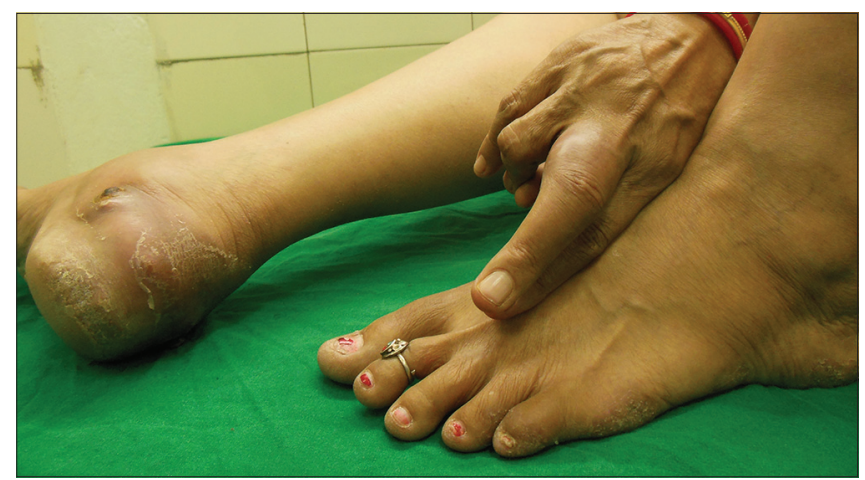

Figure 2: Irregular warty plaques with scaling over lateral aspect of bilateral soles. composed of epitheloid cells and Langhan's type of giant cells without any necrosis (Figs. 4a and 4b). A diagnosis of Tuberculous gumma with Tuberculosis verrucosa cutis was made and ATT was started.

\section{DISCUSSION}

Tuberculosis verrucosa cutis (TBVC) is warty form of cutaneous tuberculosis and was first described as "Prosecutor's wart" by Rene Laennec. It is caused by exogenous inoculation mostly due to trauma in presensitized persons with moderate to high degree of cell mediated immunity. Clinically, TBVC lesions are mainly distributed over the trauma prone areas like extremities and present as a slowly growing, asymptomatic papule/nodule to a firm verrucous plaque. Histopathologically well formed classical tubercular granuloma with pseudoepitheliomatous hyperplasia of epidermis is seen. But caseous necrosis is a rare finding [5]. Tuberculous gumma, also known as metastatic tubercular abscess is a rare form of cutaneous TB, caused by hematogenous spread of the bacilli from a primary underlying focus during periods of lowered immunity. Gumma presents clinically as cutaneous and subcutaneous nodules and/or abscesses, which are nontender and fluctuant, forming undermined ulcers on rupturing. Epitheloid

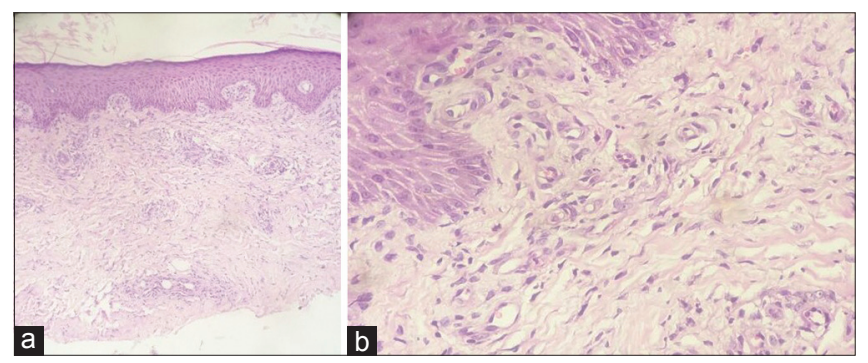

Figure 3: (a) Multiple granulomas and focal collection of inflammatory cells in the dermis [H\&E, x4], (b) Multiple granulomas composed of epitheloid cells, histiocytes, lymphocytes, Langhan's type of giant cells with small areas of necrosis [H\&E, x40].

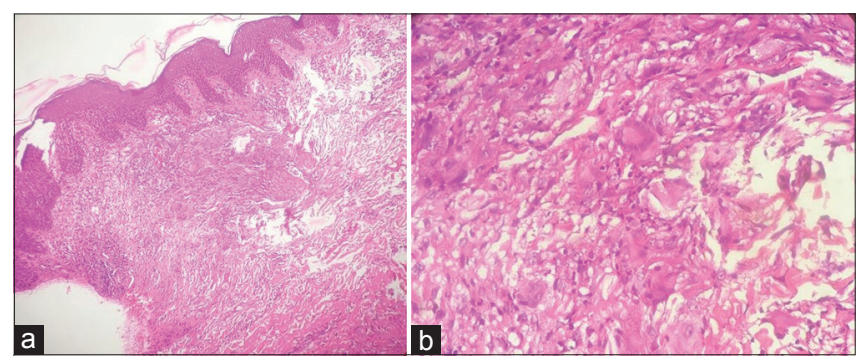

Figure 4: (a) Hyperkeratosis, acanthosis and dense inflammatory infiltrate with giant cells in the dermis [H\&E, x4], (b) Well defined granulomas composed of epitheloid cells \& Langhan's type of giant cells without any necrosis [H\&E, x40]. 
cell granulomas with extensive areas of caseous necrosis are histopathological findings of tuberculous gumma. Since, tuberculous gumma spreads hematogenously from an underlying focus in an immounocompromised patient, almost all patients presenting with gumma have systemic tubercular involvement as well as some form of immunosuppression [6]. But our patient presented without any systemic involvement or immunosuppression. Co-existence of various types of cutaneous TB has been reported in literature including Scrofuloderma with TBVC and Scrofuloderma with TB gumma $[3,4]$. To the best of our knowledge simultaneous occurrence of TB gumma and TBVC in a single patient (which occupy two extreme poles in the immunological spectrum of cutaneous TB) has not been reported till date. Hence we, report this rare case of co-existence of TBVC and TB gumma in an otherwise immunocompetent patient with no other systemic involvement.

\section{CONCLUSION}

Simultaneous occurrence of TB gumma and TBVC are extremely rare findings in an immunocompetent indivisual.

\section{Consent}

The examination of the patient was conducted according to the Declaration of Helsinki principles.

\section{REFERENCES}

1. Yates VM. Mycobacterial infections. In: Burns T, Breathnach S, Cox N, Griffith C, editors. Rook's Textbook of Dermatology. $8^{\text {th }}$ ed. Massachusettes: Wiley-Blackwell; 2010. p. 31.10

2. Kalaria VG1, Kapila R, Schwartz RA. Tuberculous gumma (cutaneous metastatic tuberculous abscess) with underlying lymphoma. Cutis. 2000;66:277-9.

3. Rao AG. Scrofuloderma associated with tuberculosis verrucosa cutis. Indian J Dermatol Venereol Leprol. 2014;80:76-8.

4. Rambhia KD, Kharkar V, Mahajan S, Khopkar US. Multifocal tuberculous gummas and bilateral scrofuloderma followed by papulonecrotic tuberculids developing during anti-tubercular therapy. Indian J Dermatol Venereol Leprol. 2016;82-4,424-6.

5. Chopra D, Chopra V, Sharma A, Chopra S, Aggarwal S, Goyal D. Unusual sites of cutaneous tuberculosis: a report of two cases. Case Rep Dermatol Med. 2017;2017:7285169.

6. Dash M, Sarangi R, Panda M. Generalized tuberculous gumma. Indian Pediatr. 2012;49:773.

Copyright by Nishant Agarwala, et al. This is an open-access article distributed under the terms of the Creative Commons Attribution License, which permits unrestricted use, distribution, and reproduction in any medium, provided the original author and source are credited.

Source of Support: Nil, Conflict of Interest: None declared. 